\title{
Estimativa de Conjuntos Atratores de Sistemas Chaveados Contínuos no Tempo Através de uma Lei de Chaveamento Mista
}

\author{
Michele C. Valentino, Flávio A. Faria, \\ Departamento de Economia, UFJF \\ 35010-000, Governador Valadares, MG \\ E-mail: michele.valentino@ufj.edu.br, flaviof15@yahoo.com.br,
}

Vilma A. Oliveira

USP - Departamento de Engenharia Elétrica

Campus São Carlos

13566-590, São Carlos, SP

E-mail:vilma@sc.usp.br.

\begin{abstract}
Resumo: $O$ objetivo deste trabalho é obter uma lei de chaveamento mista, ou seja, uma lei dependente do estado e também do tempo, a qual garante estimativas de conjuntos atratores de sistemas chaveados contínuos no tempo. Esse resultado explora um sistema formado pela combinação convexa de todos os subsistemas para estudar o comportamento assintótico da solução do sistema chaveado e suas principais características são que as estimativas de atratores podem ser encontradas mesmo na presença de subsistemas que não são ultimamente limitados e a derivada da função auxiliar, a qual desempenha o mesmo papel que a função de Lyapunov, pode assumir valores positivos em conjuntos limitados.
\end{abstract}

Palavras-chave: Sistemas chaveados, Lei de chaveamento mista, Conjuntos Atratores, Solução de Krasowski

\section{Introdução}

Sistemas chaveados surgem na prática na modelagem de operação de muitos sistemas de engenharia [1,5]. Embora chaveamento não seja um novo conceito em engenharia, na última década a teoria de sistemas chaveados tem atraído a atenção de muitos pesquisadores. Como consequência, resultados para a estabilidade e estabilização para essa classe de sistemas foi significativamente desenvolvida.

Apesar dos importantes avanços na teoria de estabilidade, os atratores de muitos sistemas chaveados podem não ser um ponto de equilíbrio. Um exemplo clássico é o sistema de controle de temperatura on-off. Para essa classe de problemas, o interesse não é estudar a estabilidade de um ponto particular de equilíbrio mais o comportamento assintótico das soluções.

O princípio de invariância é uma poderosa ferramenta para analisar o comportamento assintótico das soluções de sistemas dinâmicos. Este resultado baseia-se na existência de uma função do tipo Lyapunov para analisar o comportamento assintótico das soluções do sistema. Uma propriedade chave dessa função, é a não-positividade de sua derivada ao longo das soluções do sistema chaveado [3]. Encontrar tal função, satisfazendo todas as suposições do princípio de invariância pode ser difícil para muitos sistemas dinâmicos. Portanto, uma extensão do princípio de invariância, a qual permite a derivada de uma função $V$ ser positiva sobre alguns conjuntos, foi proposta para sistemas chaveados em [7]. Em [7], as estimativas de atratores e suas respectivas áreas de atração são encontradas apenas quando todos os subsistemas são ultimamente limitados. Para superar esta dificuldade. Em [8], foi proposto uma extensão do princípio de in- 
variância para sistemas chaveados fuzzy T-S. Este resultado explora um sistema auxiliar formado pela combinação convexa de todos os subsistemas para estudar o comportamento assintótico da solução. O objetivo deste trabalho é explorar os resultados obtidos em [8] para obter estimativas de conjuntos atratores de sistemas chaveados. A principal característica desse novo resultado é que as estimativas dos conjuntos atratores podem ser obtidas mesmo na presença de subsistemas que não são ultimamente limitados. E então, o comportamento assintótico da solução do sistema chaveado pode ser estudado para uma classe maior de sistemas do que nos resultados obtidos em [7].

\section{Conceitos Fundamentais}

Considera-se a família $\mathcal{F}=\left\{f_{p}\right\}_{p \in \mathcal{P}}$ de campos vetoriais de classe $C^{1}$, completos do $\mathbb{R}^{n}$ e a seguinte classe de sistemas chaveados contínuos no tempo

$$
\dot{x}(t)=f_{\sigma(.)}(x(t)), \quad x(0)=x_{0}
$$

em que $\mathcal{P}=\{1,2, \ldots, N\}$ é um conjunto de índices finito, $N$ é o número de subsistemas, $x(t) \in \mathbb{R}^{n}$ é o vetor do estado, $\sigma$ é uma função constante por partes chamada de lei de chaveamento, a qual pode depender do tempo $\sigma: I \rightarrow \mathcal{P}$ em que $I=\left[0, t_{f}\right)$ com $0<t_{f} \leq \infty$, ou do estado $\sigma: X \rightarrow P \operatorname{com} X \subseteq \mathbb{R}^{n}$. Uma função contínua suave por partes $x_{\sigma(.)}(t): I \rightarrow \mathbb{R}^{n}$ é uma solução do sistema chaveado (1) no intervalo $I$ se $x_{\sigma(.)}(t)$ satisfaz $\dot{x}_{\sigma(.)}(t)=f_{p}\left(x_{\sigma(.)}(t)\right)$ para todo $t$ tal que $\sigma()=$.$p . Se f(x)=f_{\sigma(x)}(x)$ é um campo vetorial mensurável e localmente limitado do $\mathbb{R}^{n}$ diz-se que uma curva absolutamente continua $x(t): \mathcal{I} \rightarrow \mathbb{R}^{n}$ é uma solução de Krasowski de (1) no intervalo $\mathcal{I}$ se satisfaz a inclusão diferencial

$$
\dot{x} \in K f(x(t))
$$

para todo $t \in \mathcal{I}$, com $K f(x)=\bigcap_{\delta>0} \overline{c o}\{f(B(x, \delta))\}$ em que $\overline{c o}$ é o fecho do envoltório convexo e $B(x, \delta)$ é a bola aberta de raio $\delta$ e centro $x$. Note que $f_{\sigma(.)}(x)$ é mensurável se e somente se $\sigma($. é mensurável. Portanto, se $\sigma($.$) é mensurável, então para qualquer condição inicial x_{0}$ existe a solução de Krasowski local do sistema (1). Denota-se $\varphi_{\sigma(.)}\left(t, x_{0}\right)$, ou simplesmente $\varphi\left(t, x_{0}\right)$, a solução de (1) iniciando em $x_{0}$ no tempo $t=0$ através da lei de chaveamento $\sigma($.$) .$

Neste trabalho, estuda-se soluções do sistema (1) através de uma classe particular de lei de chaveamento, então algumas definições preliminares e proposições $[3,4,6]$ as quais serão usadas no desenvolvimento do resultado principal são apresentadas.

Definição .1 Seja $H$ um conjunto fechado e invariante para o sistema (1) através da lei de chaveamento $\sigma$. Diz-se que $H$ é um atrator se existe uma vizinhança $U$ de $H$ tal que, para toda condição inicial $x_{0} \in U, \varphi\left(t, x_{0}\right) \rightarrow H$ quando $t \rightarrow+\infty$.

Definição .2 (Invariância Fraca) Um conjunto $\mathcal{N}$ é fracamente invariante com respeito ao sistema chaveado (1) se para cada $x_{0} \in \mathcal{N}$ existe um indice $p \in \mathcal{P}$, uma solução $\varphi\left(t, x_{0}\right)$ do campo vetorial $f_{p}(x)$ e um número real $b>0$ tal que $\varphi\left(t, x_{0}\right)$ pertence ao conjunto $\mathcal{N}$ para qualquer $t \in[-b, 0]$ ou $t \in[0, b]$.

Definição .3 (Pontos Limites) Um ponto $q \in \mathbb{R}^{n}$ é um ponto limite de $\varphi\left(t, x_{0}\right)$ se existe uma sequência de tempos de chaveamentos $\left\{t_{k}\right\}_{k \in N}$, com $t_{k} \rightarrow \infty$, quando $k \rightarrow \infty$ tal que $\lim _{k \rightarrow \infty} \varphi\left(t_{k}, x_{0}\right)=q$. O conjunto de todos os pontos limites de $\varphi\left(t, x_{0}\right)$ é denotado por $\omega\left(x_{0}\right)$.

Proposição .1 (Propriedades de Conjunto Limite) Seja $\varphi\left(t, x_{0}\right) \in \mathcal{S}_{\text {dwell }}$ uma solução chaveada limitada de (1) para $t \geq 0$. Então, $\omega\left(x_{0}\right)$ é não vazio, compacto e fracamente invariante. Além disso, $\varphi\left(t, x_{0}\right)$ é atraído para $\omega\left(x_{0}\right)$. 
Demonstração .1 Veja [2].

Na próxima seção, é apresentado o resultado principal, o qual fornece estimativa de conjuntos atratores de sistemas chaveados. A lei de chaveamento que garante essa estimativa pode depender do tempo ou do estado e esta escolha depende do conjunto onde a solução se encontra.

\section{Estimativas de Conjuntos Atratores de Sistemas Chaveados}

Nesta seção, o Teorema 1 de [8] é explorado para obter estimativas de conjunto atrator de sistemas chaveados. Neste resultado, um sistema auxiliar formado pela combinação convexa de todos os subsistemas, é usado para estudar o comportamento assintótico das soluções do sistema chaveado. A vantagem dessa estratégia é que as estimativas dos conjuntos atratores são obtidas mesmo na presença de subsistemas que não são ultimamente limitados.

Seja o conjunto

$$
M=\left\{\alpha=\left(\alpha_{1}, \alpha_{N}\right) \in \mathbb{R}^{N}: \alpha_{p} \geq 0, \forall p \in \mathcal{P} \text { and } \sum_{p=1}^{N} \alpha_{p}=1\right\}
$$

com constantes $\alpha_{p}$ definindo a combinação convexa dos subsistemas $f_{p}$ para todo $p \in \mathcal{P}$.

Considere o sistema auxiliar

$$
\dot{x}(t)=\sum_{p=1}^{N} \alpha_{p} f_{p}(x(t))=f(x(t))
$$

em que $\alpha \in \mathcal{M}$. Seja $V: \mathbb{R}^{n} \rightarrow \mathbb{R}$ uma função de classe $C^{1}$ e defina os seguintes conjuntos ao longo da solução do sistema (3):

$$
\begin{aligned}
C_{\alpha} & =\left\{x \in \mathbb{R}^{n}: \nabla V(x) f(x) \geq 0\right\}, \\
\Omega_{v} & =\left\{x \in \mathbb{R}^{n}: V(x) \leq v\right\} \\
\Gamma_{p} & =\left\{x \in \mathcal{B}: \nabla V(x) f_{p}(x)<0\right\} .
\end{aligned}
$$

$\operatorname{com} \mathcal{B} \subseteq \mathbb{R}^{n}$.

A próxima proposição fornece uma lei de chaveamento dependente do estado que garante que a derivada da função $\mathrm{V}$ decresce ao longo da solução do sistema chaveado (1) e é usada na demonstração do resultado principal.

Proposição .2 Sejam $\mathcal{B}$ um subconjunto do $\mathbb{R}^{n}, V: \mathbb{R}^{n} \rightarrow \mathbb{R}$ uma função de classe $\mathcal{C}^{1} e$ considere $\alpha \in \mathcal{M}$ tal que

$$
\frac{\partial V}{\partial x}\left[\sum_{p=1}^{N} \alpha_{p} f_{p}(x)\right]<0
$$

para todo $t$ enquanto $x(t) \in \mathcal{B}$. Então, existe uma lei de chaveamento $\sigma(x)$, a qual assegura que a função $V$ decresce ao longo da solução do sistema chaveado (1) em $\mathcal{B}$. Mais ainda, se a lei de chaveamento é mensurável, então $V$ decresce ao longo da solução de Krasowski de (1) em $\mathcal{B}$.

Demonstração .2 Seguindo [4], sejam $\mathcal{B}$ um subconjunto do $\mathbb{R}^{n}, V$ uma função de classe $\mathcal{C}^{1}$ e considere $\alpha \in \mathcal{M}$, então para todo $t$ enquanto $x(t) \in \mathcal{B}$ existe pelo menos um $p \in \mathcal{P}$ tal que

$$
\frac{\partial V}{\partial x}\left[f_{p}(x)\right]<0 .
$$

Portanto, existe uma lei de chaveamento $\sigma(x)$, tal que $V$ decresce ao longo da solução do sistema chaveado (1) em $\mathcal{B}$ enquanto a solução existir. Caso a lei de chaveamento seja mensurável, então para toda condição inicial $x_{0} \in \mathcal{B}$ pode-se assegurar a existência local da solução de Krasowski de (1) e um $p \in \mathcal{P}$ tal que (5) seja satisfeita, então a derivada de $V$ decresce ao longo dessa solução de Krasowski de (1) em $\mathcal{B}$ enquanto a solução existir. 
Seguindo os mesmos passos do Teorema 2.5 de [3], uma lei de chaveamento mensurável $\sigma: \mathcal{B} \rightarrow \mathcal{P}$ satisfazendo (5), pode ser definida da forma:

$$
\sigma(x)=\left\{\begin{array}{lc}
1, & \text { se } \quad x \in \Gamma_{1} \\
p, & \text { se } \quad x \in\left(\Gamma_{p} \backslash\left(\bigcup_{k<p} \Gamma_{k}\right)\right)
\end{array}\right.
$$

ou equivalentemente $\sigma(x)=\min \left\{p: \nabla V(x) f_{p}(x)<0\right\}$. De fato, considere $\mathcal{B}=\mathbb{R}^{n} \backslash \Omega_{\ell}$, a função $\sigma: \mathcal{B} \rightarrow \mathcal{P}$ dada por lei e $F_{p}=\{x \in \mathcal{B}: \sigma(x)=p\}$ o qual satisfaz $F_{i} \cap F_{j}=\emptyset$ sempre que $i \neq j \in \mathcal{P}$ e $\bigcup_{p \in \mathcal{P}} F_{p}=\mathcal{B}$. Sejam as $\sigma$-álgebra $\Sigma_{1}$ de Borel e $\Sigma_{2}$ formada pelo conjuntos das partes de $\mathcal{P}$, então

$$
\sigma^{-1}(\{p\})=\left(\Gamma_{p} \backslash\left(\bigcup_{k<p} \Gamma_{k}\right)\right)=F_{p}
$$

Como $\Gamma_{p} \in \Sigma_{1}$ e $F_{p}=\left(\bigcup_{k<p} \Gamma_{k}\right)^{c} \bigcap \Gamma_{p} \in \Sigma_{1}$ para todo $p \in \mathcal{P}$, então $\sigma(x)$ é uma função mensurável.

No próximo teorema é apresentado o resultado principal deste trabalho e considera-se que $\mathcal{B}=\overline{\mathbb{R}^{n} \backslash \Omega_{\ell}}$. A lei de chaveamento desse resultado depende do estado fora do conjunto $\mathcal{B}$ e do tempo dentro desse conjunto.

Teorema .1 Seja $V(x): \mathbb{R}^{n} \rightarrow \mathbb{R}$ uma função de classe $\mathcal{C}^{1}$ e considere $\alpha \in M$. Se existe um $\ell \in \mathbb{R}$ satisfazendo $\sup _{x \in C_{\alpha}} V(x)<\ell<\infty$ tal que $\Omega_{\ell}$ é limitado, então toda solução limitada $\varphi\left(t, x_{0}\right)$ de (1) possuindo uma lei de chaveamento dwell-time em $\Omega_{\ell}$ e uma lei de chaveamento mensurável $\sigma(x)$ satisfazendo a Condição 1 para todo $t$ enquanto $x(t) \in \overline{\Omega_{\ell}^{c}}$ é atraída para um conjunto fracamente invariante em $\Omega_{\ell}$. Portanto, para qualquer condição inicial $x_{0}$, a solução é atraída para um conjunto fracamente invariante em $\Omega_{\ell}$ e então, $\Omega_{\ell}$ é uma estimativa do conjunto atrator de (1).

Demonstração .3 Seja $\left[0, t_{f}\right)$ o intervalo maximal de existência da solução chaveada.

Primeiramente, considere $x_{0} \in \Omega_{\ell}$ e $\varphi\left(t, x_{0}\right)$ sendo uma solução de (1) possuindo um chaveamento dwell-time em $\Omega_{\ell}$ e satisfazendo a Condição 1 para todo $x \in \overline{\Omega_{\ell}^{c}}$. Suponha a existência de um $\tau>0$ tal que $\varphi\left(\tau, x_{0}\right) \notin \Omega_{\ell}$. Então, existe $\bar{\tau} \in(0, \tau)$ tal que $V\left(\varphi\left(\bar{\tau}, x_{0}\right)\right)=\ell$ (pela continuidade de $V$ e $\left.\varphi\left(t, x_{0}\right)\right)$ e $V\left(\varphi\left(t, x_{0}\right)\right)>\ell, \forall t \in(\bar{\tau}, \tau]$. Como a Condição 1 é satisfeita fora de $\Omega_{l}$, então $\frac{\partial V}{\partial x}[f(x(t))]<0$ para todo $x \in \overline{\Omega_{\ell}^{c}}$. Portanto, $V$ decresce ao longo da solução chaveada de (1) em $\overline{\Omega_{\ell}^{c}}$ levando a uma contradição, assim $\varphi\left(t, x_{0}\right) \in \Omega_{\ell}$ para todo $t \in\left[0, t_{f}\right)$. Uma vez que a solução limitada é dweel-time então $t_{f}=+\infty$ e portanto, $\varphi\left(t, x_{0}\right) \in \Omega_{\ell}$ para todo $t \geq 0$. Finalmente, pela Proposição 1, para toda solução limitada $\varphi\left(t, x_{0}\right), \omega\left(x_{0}\right)$ é não vazio, compacto, fracamente invariante e $\omega\left(x_{0}\right) \subset \Omega_{\ell}$. Mais ainda, $\varphi\left(t, x_{0}\right)$ é atraída para $\omega\left(x_{0}\right)$. Então, a solução é atraída para um conjunto fracamente invariante dentro do conjunto $\Omega_{\ell}$.

Agora, considere $x_{0} \notin \Omega_{\ell}$ e $\varphi\left(t, x_{0}\right)$ sendo uma solução limitada de (1) através de uma lei de chaveamento mensurável satisfazendo a Condição 1. Então pela Proposição 2, a função $V$ decresce ao longo da solução de Krasowski de (1) para todo $t \in\left[0, t_{f}\right)$ enquanto $x \in \overline{\Omega_{\ell}^{c}}$. Uma vez que a solução é limitada então $t_{f}=+\infty$, portanto existe um tempo $t_{1}$ tal que $V\left(x\left(t_{1}\right)\right)<\ell$ e então o resultado segue da primeira parte desta demonstração.

Portanto, o conjunto limitado $\Omega_{\ell}$ é a estimativa de um conjunto atrator do sistema chaveado, pois para qualquer condição inicial $x_{0}$ a solução é atraída para um conjunto fracamente invariante em $\Omega_{\ell}$. 
Exemplo .1 Considere um sistema chaveado com $\mathcal{P}=\{1,2\}$ e

$$
f_{1}(x)=\left[\begin{array}{c}
-2 x_{1}^{3}-2 x_{1} x_{2}^{2}+x_{2} \\
2 x_{2}-x_{1}
\end{array}\right], \quad f_{2}(x)=\left[\begin{array}{c}
2 x_{1}-x_{2} \\
-2 x_{1}^{2} x_{2}-2 x_{2}^{3}+x_{1}
\end{array}\right]
$$

Seja $V(x)=\frac{x_{1}^{2}+x_{2}^{2}}{2}$ e $\alpha_{1}=\alpha_{2}=0.5$. Então

$$
\begin{aligned}
\nabla V(x) f(x) & =x_{1}\left(-x_{1}^{3}-x_{1} x_{2}^{2}+0.5 x_{2}+x_{1}-0.5 x_{2}\right) \\
& +x_{2}\left(x_{2}-0.5 x_{1}-x_{1}^{2} x_{2}-x_{2}^{3}+0.5 x_{1}\right) \\
& =\left(x_{1}^{2}+x_{2}^{2}\right)\left(-x_{1}^{2}-x_{2}^{2}+1\right) .
\end{aligned}
$$

Portanto, $C_{\alpha}=\left\{x \in \mathbb{R}^{2}: x_{1}^{2}+x_{2}^{2} \leq 1\right\}$ e $\ell>0.5$. Note que o conjunto onde a derivada (8) assume valor zero está em $\Omega_{\ell}$, o qual é limitado. Assim pelo Teorema .1, qualquer solução limitada $\varphi\left(t, x_{0}\right)$ possuindo uma lei de chaveamento dwell-time em $\Omega_{\ell}$ e uma lei $\sigma(x)$ satisfazendo Condição 1 para todo $x \in \overline{\Omega_{\ell}^{c}}$ é atraída para um conjunto fracamente invariante em $\Omega_{\ell} . A$ Figura 1(a) ilustra a simulação no domínio de tempo para $x_{0}=[-2-2.2]$, a Figura 1(b) mostra a lei de chaveamento $\sigma($.$) e a Figura 2(b)$ mostra que a função $V$ decresce fora de $\Omega_{\ell}$, isto é, a lei de chaveamento $\sigma($.$) satisfaz a Condição 1$ para todo $x \in \overline{\Omega_{\ell}^{c}}$. Essa simulação confirma o resultado do Teorema 1 por mostrar um conjunto atrator dentro do círculo de raio $\sqrt{1.2}$ para $\ell=0.6$ (Figura $2(a)$ ).
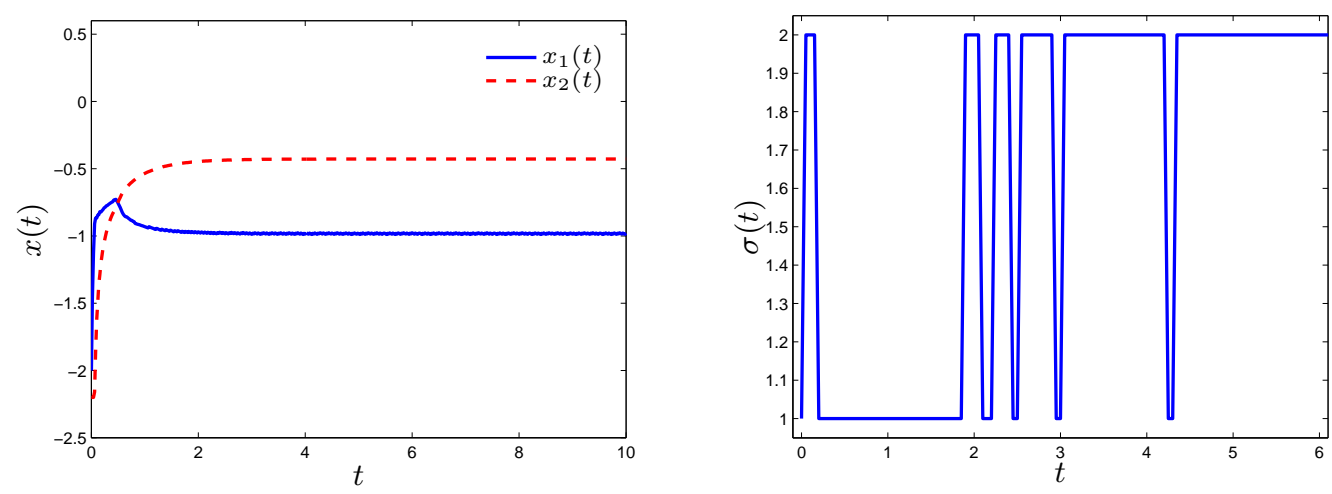

Figura 1: a) Solução chaveada para a condição inicial $x_{0}=[-2-2.2]$ para o Exemplo .1 (b) chaveamento $\sigma(t)$ para o Exemplo .1
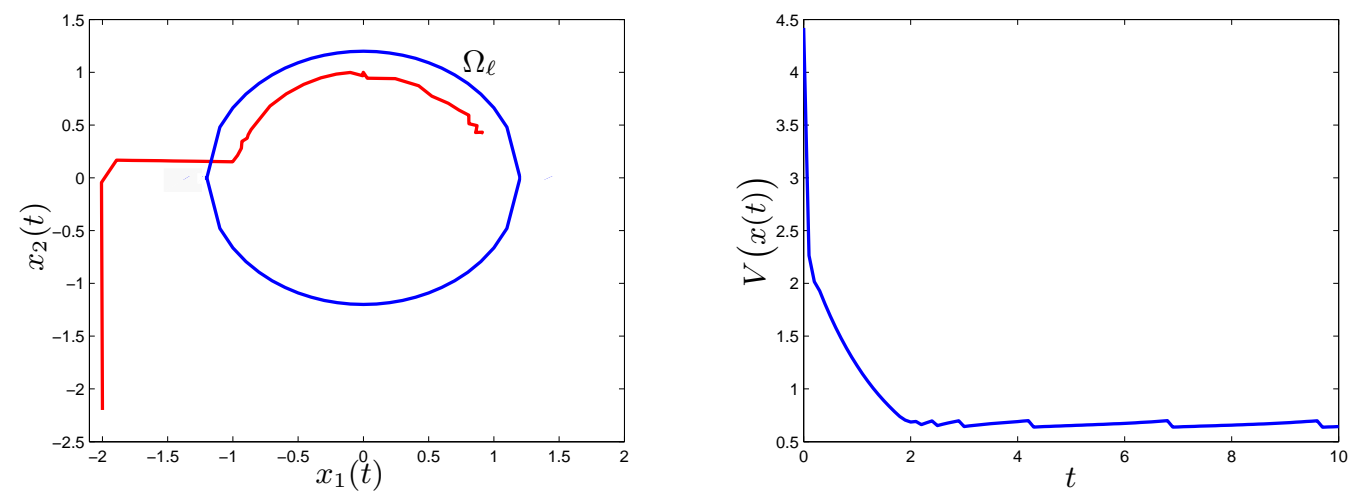

Figura 2: a) Plano de fase com $x_{0}=\left[\begin{array}{ll}-2 & -2.2\end{array}\right]$ para o Exemplo .1 (b) função $V$ ao longo da solução do sistema chaveado para o Exemplo .1 


\section{Referências}

[1] Arrifano, N. S. D.; Oliveira, V. A.; Ramos, R. A.; Bretas S, N. G. E Oliveira, R. V., Fuzzy stabilization of power systems in a co-generation scheme subject to random abrupt variations of operating conditions, IEEE Transactions on Control System Technology v.15, n.2, pp.384-393, 2007.

[2] A. Bacciotti e L. Mazzi, An invariance principle for nonlinear switched systems, Systems and Control Letters v.54, n.11, p.1109-1119, 2005.

[3] A. Bacciotti e L. Mazzi, From artstein-sontag theorem to the min-projection strategy, Transactions of the Institute of Measurement Control v.32, n.6, p.571-581, 2010.

[4] J. S. Chiou, C. J. Wang, C. M. Cheng e C. C. Wang, Analysis and synthesis of switched nonlinear systems using the ts fuzzy model, Applied Mathe- matical Modelling v.34, n.6, p.1467-1481, 2010.

[5] Deaecto, G. S., " Projeto de controladores dinâmicos com comutação", Dissertação de Mestrado, Departamento de Engenharia Elétrica e de Computação, Universidade Estadual de Campinas, Campinas, Brasil, 2010.

[6] D. Liberzon, "Switching in Systems and Control", BIRKHAUSER, Boston, USA, 2003.

[7] Valentino, M. C.; Oliveira, V. A.; Alberto, L. F. E SantAnna, D. A., An extension of the invariance principle for dwell-time switched nonlinear systems, Systems \& Control Letters v.61, n.4, p.580 - 586, 2012.

[8] Michele C. Valentino, Flávio A. Faria e Vilma A. Oliveira (2013). An extension of the invariance principle for switched T-S fuzzy systems synthesis of switched nonlinear systems using the ts fuzzy model, em "ECC- 3 European Control Conference" pp.4322-4327, Zürich, Switzerland, 2013. 